EPJ Web of Conferences 71, 00141 (2014)

DOI: $10.1051 /$ epjconf/20147100141

(C) Owned by the authors, published by EDP Sciences, 2014

\title{
Measurement of properties of the Higgs boson in the diphoton channel using the ATLAS detector
}

\author{
Hongtao YANG ${ }^{1, a}$ On behalf of the ATLAS Collaboration \\ ${ }^{1}$ Department of Physics, University of Wisconsin-Madison, 1150 University Avenue, Madison, WI 53706- \\ 1390, USA
}

\begin{abstract}
Results for the properties of the Higgs boson measured in the diphoton decay channel by the ATLAS experiment at the LHC are presented, which include coupling, spin, and differential cross section measurements. Searches for $t \bar{t} H$ production and Flavor Changing Neutral Current $t \rightarrow c H$ decay in this channel are also briefly discussed. The results are based on either the entire data sample collected with the ATLAS detector in 2011 at $\sqrt{s}=7 \mathrm{TeV}$ and 2012 at $\sqrt{s}=8 \mathrm{TeV}$, or only the entire $8 \mathrm{TeV}$ data sample. Within the experimental and theoretical uncertainties, no significant deviation from the Standard Model expectation is observed.
\end{abstract}

\section{Introduction}

According to the Standard Model (SM) of particle physics, elementary particles acquire their masses through a mechanism that spontaneously breaks the electroweak symmetry. A consequence of this model is the existence of a scalar particle, the SM Higgs boson. In July 2012, the ATLAS and CMS collaborations independently reported observations of a new particle, using $p p$ collisions from the 2011 and 2012 datasets collected at the LHC until the end of June 2012. While it seems to be compatible with the SM Higgs boson, more detailed and precise measurements of the properties of this new particle are essential to further elucidate its role in electroweak symmetry breaking (EWSB) and mass generation. The diphoton decay channel is of great importance for this purpose because it has good mass resolution and is sensitive to the existence of new physics due to loop involved in this decay mode.

The Higgs boson property measurements in the diphoton decay channel reported here are obtained from either the whole data sample collected with the ATLAS detector [1] in 2011 at $\sqrt{s}=7 \mathrm{TeV}$ and 2012 at $\sqrt{s}=8 \mathrm{TeV}$, or only the whole $8 \mathrm{TeV}$ data sample ${ }^{1}$.

This article is organized as follows: The observation and mass measurement results are detailed in Section 2, followed by coupling measurement results in Section 3. The spin measurement results are presented in Section 4. The differential cross section measurements are discussed in Section 5, and results for the $t \bar{t} H$ and Flavor Changing Neutral Current (FCNC) $t \rightarrow c H$ searches are reported in Section 6. The conclusions are summarized in Section 7.

\footnotetext{
a e-mail: Hongtao.Yang@cern.ch

${ }^{1}$ The exact data sample used for each analysis will be specified before the analysis itself is described.
} 


\section{Observation and mass measurement}

\subsection{Event selection}

The observation and mass measurement results are based on $4.7 \mathrm{fb}^{-1}$ of $7 \mathrm{TeV}$ data and $20.7 \mathrm{fb}^{-1}$ of $8 \mathrm{TeV}$ data after application of data quality requirements.

In the event selection, at least two photons satisfying tight identification criteria based on the shapes of the electromagnetic showers are required. The transverse energies for the leading and subleading photons are required to be larger than $40 \mathrm{GeV}$ and $30 \mathrm{GeV}$, respectively. Both photons need to be within the fiducial calorimeter region of $|\eta|<2.37$ (excluding the transition region between the barrel and the end-cap calorimeters, $1.37<|\eta|<1.56$ ). They are also required to be isolated using criteria based on both the inner tracker and the calorimeter.

The photon energy calibration is obtained from a detailed simulation of the detector geometry and response independently for converted and unconverted photons. The calibration is refined by applying $\eta$-dependent correction factors obtained from studies of $Z \rightarrow e e$ decays in data. The mass resolution for a SM Higgs signal at $126.5 \mathrm{GeV}$ after selection is expected to be $1.8 \mathrm{GeV}$, while the mass scale uncertainty for the $126.5 \mathrm{GeV}$ signal is about $700 \mathrm{MeV}$.

\subsection{Event categorization}

The selected events from $8 \mathrm{TeV}(7 \mathrm{TeV})$ dataset are divided into 14 (10) exclusive categories based on event properties. The categories differ in signal-to-background ratio as well as invariant mass resolution and thus their use in the analysis increases the sensitivity of the measurement. Gluon fusion $(g g F)$ is the dominant production mode of Higgs bosons at the LHC, contributing $87 \%$ of the total production cross section at $m_{H}=126.5 \mathrm{GeV}$, while vector boson fusion $(V B F)$ and associated production with vector bosons $(V H)$ contribute $7 \%$ and $5 \%$, respectively. Several categories are designed to increase the sensitivity to the $V B F$ and $V H$ processes. An order of preference is applied when sorting events into categories (Figure 1).

\subsection{Signal significance}

The observed and expected local $p_{0}$ values ${ }^{2}$ are shown in Figure 2(a) as a function of $m_{H}$. The largest local significance in the combination of $7 \mathrm{TeV}$ and $8 \mathrm{TeV}$ data is found to be $7.4 \sigma$ at $m_{H}=126.5 \mathrm{GeV}$, which means that the observation of the new boson can be established by this channel alone.

\subsection{Mass measurement}

For the mass measurement, both the mass of the Higgs boson $m_{H}$ and the signal strength parameter $\mu \equiv \sigma / \sigma_{S M}$ are treated as free parameters in the fit, where the unbinned extended maximum likelihood fitting technique is used. The best-fit values of $m_{H}$ and $\mu$ are shown in Figure 2(b), where the best-fit $m_{H}$ is found to be $126.8 \mathrm{GeV}$. The statistical and systematic uncertainties on $m_{H}$ are evaluated to be $\pm 0.2 \mathrm{GeV}$ and $\pm 0.7 \mathrm{GeV}$, respectively. The dominant contribution to the systematic uncertainty comes from the uncertainties on the photon energy scale. A combination with the channel $H \rightarrow Z Z^{*} \rightarrow 4 l$ yields a measured mass of $m_{H}=125.5 \pm 0.2$ (stat) ${ }_{-0.6}^{+0.5}$ (syst) $\mathrm{GeV}$.

\footnotetext{
${ }^{2}$ The local $p_{0}$ value is the probability of the background fluctuating beyond the observation in the data at a particular $m_{H}$.
} 


\section{ICNFP 2013}

\section{ATLAS Preliminary}

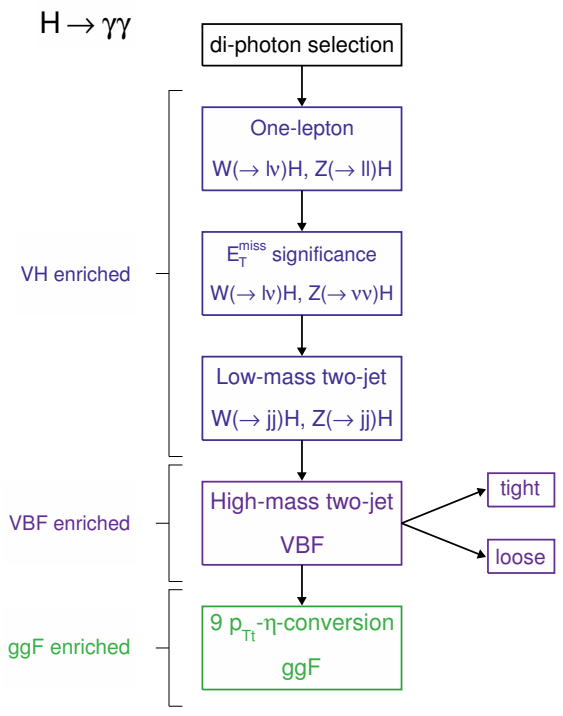

Figure 1. Flow-chart of the event categorization, giving the order of selection of the different categories [2].

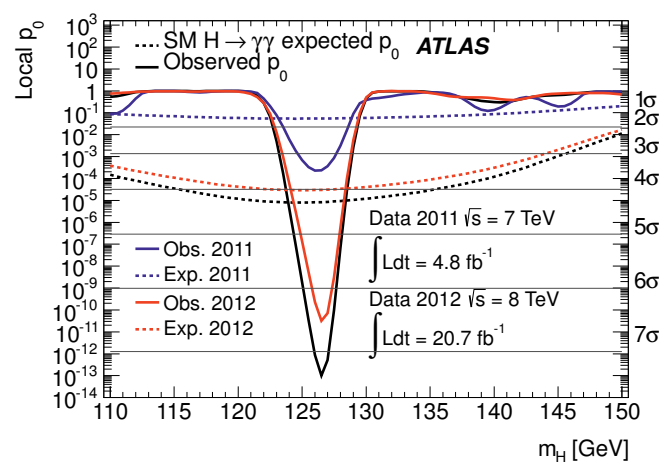

(a)

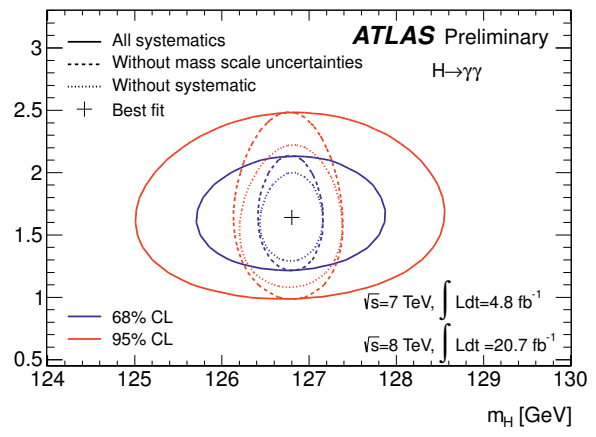

(b)

Figure 2. (a) Observed local $p_{0}$ as a function of the Higgs boson mass $m_{H}$ for the $7 \mathrm{TeV}$ data (blue), the $8 \mathrm{TeV}$ data (red) and their combination (black). The dashed curves show the expected median local $p_{0}$ for the SM Higgs boson hypothesis when tested at a given $m_{H}$ [3]. (b) The best-fit values of $m_{H}$ and $\mu$, and their $68 \%$ (blue) and 95\% (red) confidence level contours. Results when photon energy scale systematic uncertainties are removed (dashed), and results when all systematic uncertainties are removed (dotted), are also shown [2].

\section{Coupling measurements}

The coupling measurement results are based on $4.7 \mathrm{fb}^{-1}$ of $7 \mathrm{TeV}$ data and $20.7 \mathrm{fb}^{-1}$ of $8 \mathrm{TeV}$ data after application of data quality requirements. The event selection and event categorization used here are the same as those described in Sections 2.1 and 2.2. 


\subsection{Signal strength}

At $m_{H}=125.5 \mathrm{GeV}$, the signal strength parameter for inclusive Higgs boson production $\mu$ is found to be 1.55 . The uncertainty on it can be broken down to ${ }_{-0.22}^{+0.23}$ (stat), ${ }_{-0.13}^{+0.17}$ (syst) and ${ }_{-0.12}^{+0.17}$ (theory). The systematic uncertainty includes contribution from uncertainties which can affect the signal yield, the signal resolution, and the migration of events between categories. The theory uncertainty includes the uncertainties on the Higgs boson production cross section, on the choice of parton distribution function (PDF) set, and on the decay branching ratio.

The compatibility in the signal strength parameter between the data and the SM Higgs boson signal plus background hypothesis is estimated to be at $1.9 \sigma$ level.

\subsection{Couplings and production modes}

As briefly mentioned in Section 2.2, several categories targeting different production modes have been introduced. For the $8 \mathrm{TeV}$ data sample, two of them (loose and tight high-mass two-jet) are enriched in $V B F$ events, and three of them (low-mass two-jet, $E_{\mathrm{T}}^{\text {miss }}$ significance and one-lepton) are enriched in $V H$ events. For $7 \mathrm{TeV}$ there is one category enriched in $V B F$ events. Such an analysis provides constraints on the signal strength of individual production modes.

The signal strength parameters for different Higgs boson production modes are introduced to characterize their contributions to the observed excess. To further enhance the sensitivity, the $g g F$ and $t \bar{t} H$ processes are grouped together to share the same signal strength parameter, $\mu_{g g F+t \bar{t} H}$, as they both involve the coupling between a Higgs boson and top quarks. Similarly, the $V B F$ and $V H$ processes are grouped together to share the same signal strength parameter $\mu_{V B F+V H}$. The fitted $\mu_{g g F+t \bar{t} H}$ and $\mu_{V B F+V H}$ are multiplied by a common scale factor $B / B_{\mathrm{SM}}$, where $B$ is the branching ratio for $H \rightarrow \gamma \gamma$ and $B_{\mathrm{SM}}$ is the branching ratio for $H \rightarrow \gamma \gamma$ predicted by the SM. The simultaneously fitted values of $\mu_{g g F+t \bar{t} H} \times B / B_{\mathrm{SM}}$ and $\mu_{V B F+V H} \times B / B_{\mathrm{SM}}$ in data are presented in Figure 3(a) as a red cross, where its $68 \%$ and $95 \%$ confidence level contours are also shown in red dashed lines.

A simultaneous fit is also performed to determine the signal strengths of $\mu_{g g F+t \bar{t} H} \times B / B_{\mathrm{SM}}, \mu_{V B F} \times$ $B / B_{\mathrm{SM}}$, and $\mu_{V H} \times B / B_{\mathrm{SM}}$, where the $V B F$ and $V H$ production modes are separated, and the results are summarized in Figure 3(b).

\section{Spin measurement}

The spin measurement results are based on $20.7 \mathrm{fb}^{-1} 8 \mathrm{TeV}$ data after application of data quality requirements.

In this analysis we compare the SM spin-0 hypothesis to a specific spin-2 "graviton-like" model with minimal couplings at $125.5 \mathrm{GeV}$. The spin-2 resonance can be produced either via gluon fusion $(g g)$ or via P-wave quark-antiquark annihilation $(q \bar{q})$. Five scenarios corresponding to different admixtures of the production modes are considered. The discrimination between the spin hypotheses is enhanced when the spin-2 particle is produced predominantly via gluon fusion.

In the two-body decay $H \rightarrow \gamma \gamma$, the spin information is extracted from the distribution of the polar angle $\theta^{*}$ of the photons with respect to the $z$-axis of the Collins-Soper frame, which will be defined later. This choice minimizes the expected impact of initial state radiation and achieves a better discrimination power than other choices of axis (such as the beam axis or the boost axis of the particle). 


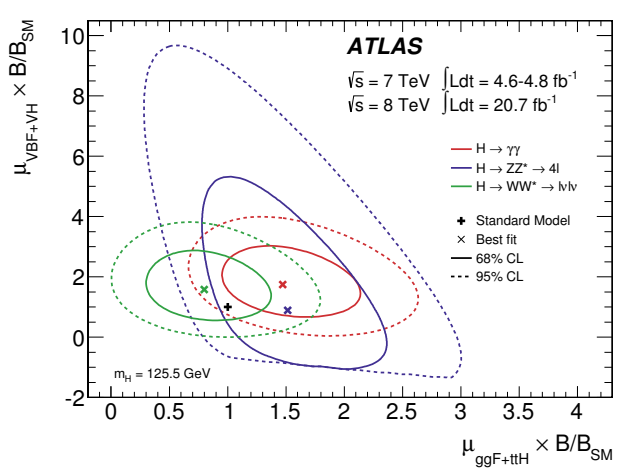

(a)

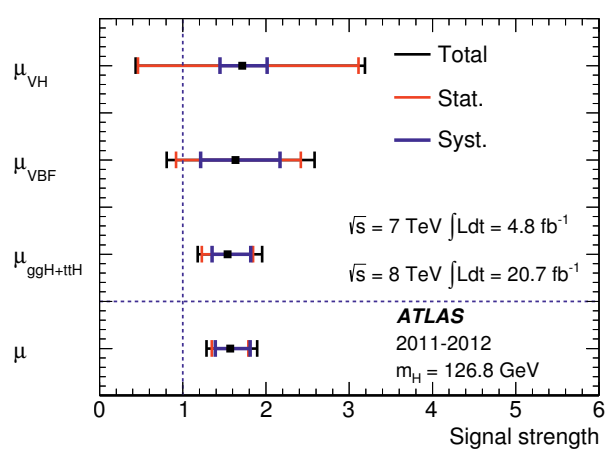

(b)

Figure 3. (a) The best-fit values ( $\times$ ) of $\mu_{g g F+t \bar{t} H} \times B / B_{S M}$ and $\mu_{V B F+V H} \times B / B_{S M}$ from a simultaneous fit to the data and their $68 \%$ (solid) and $95 \%$ (dashed) confidence level contours. Results are shown for $H \rightarrow \gamma \gamma$, $H \rightarrow Z Z^{*} \rightarrow 4 l$, and $H \rightarrow W W^{*} \rightarrow l v l v$. The expectation for a SM Higgs boson is also shown (+) [3]. (b) Measured signal strengths $\mu_{g g F+t \bar{t} H}, \mu_{V B F}$ and $\mu_{V H}$ for the different $H \rightarrow \gamma \gamma$ production modes, as well as overall strength $\mu[3]$.

\subsection{Event selection and analysis strategy}

The diphoton candidate selection used in spin analysis is close to the one outlined in Section 2.1, except that the leading and sub-leading photons are required to satisfy $p_{\mathrm{T}}^{\gamma_{1}} / m_{\gamma \gamma}>0.35$ and $p_{\mathrm{T}}^{\gamma_{2}} / m_{\gamma \gamma}>$ 0.25 , respectively, where $p_{\mathrm{T}}^{\gamma}$ is the transverse energy of a photon calculated using the vertex position. These criteria are introduced in order to minimize the correlation between $m_{\gamma \gamma}$ and $\cos \theta^{*}$ induced by the selection on the transverse momenta. The near absence of correlations when using relative $p_{\mathrm{T}}$ selection is a consequence of the $\cos \theta^{*}$ definition in the Collins-Soper frame:

$$
\cos \theta^{*}=\frac{\sinh \left(\eta_{\gamma_{1}}-\eta_{\gamma_{2}}\right)}{\sqrt{1+\left(p_{\mathrm{T}}^{\gamma \gamma} / m_{\gamma \gamma}\right)^{2}}} \cdot \frac{2 p_{\mathrm{T}}^{\gamma_{1}} p_{\mathrm{T}}^{\gamma_{2}}}{m_{\gamma \gamma}^{2}},
$$

where $p_{\mathrm{T}}^{\gamma \gamma}$ is the transverse momentum of the diphoton system. The residual correlation between $\cos \theta^{*}$ and $m_{\gamma \gamma}$ is mainly due to the $\left(p_{\mathrm{T}}^{\gamma \gamma} / m_{\gamma \gamma}\right)$ term, which is negligible except at large values of $\cos \theta^{*}$ where only very high $p_{\mathrm{T}}$ photon pairs pass the selection.

Candidates with diphoton invariant masses $\left(m_{\gamma \gamma}\right)$ in the range $105-160 \mathrm{GeV}$ are then retained. Within this range, a signal region is defined between $122-130 \mathrm{GeV}$ and sideband regions are defined as $105 \mathrm{GeV}<m_{\gamma \gamma}<122 \mathrm{GeV}$ and $130 \mathrm{GeV}<m_{\gamma \gamma}<160 \mathrm{GeV}$. The spin analysis makes use of the near absence of correlations between $m_{\gamma \gamma}$ and $\cos \theta^{*}$ to perform a fit to the product of the probability density functions of these variables in the signal region.

\subsection{Results}

The separation between the spin- 0 and spin- 2 hypotheses is maximal in the case where the spin- 2 resonance is produced by gluon fusion, where the spin- 2 hypothesis is excluded at $99.3 \%$ confidence level, as shown in Figure 4(a). Figure 4(b) shows the observed values of the test statistics and the median expected values for the spin- 0 and spin- 2 hypotheses, as a function of the fraction of $q \bar{q}$ 


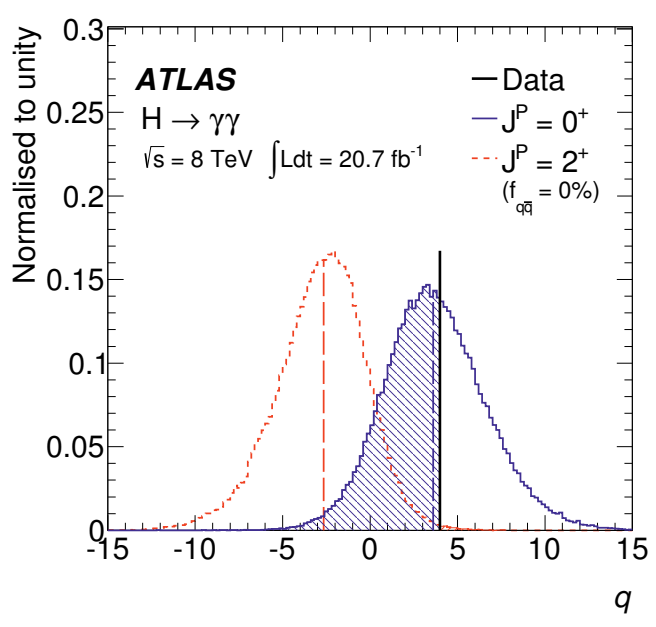

(a)

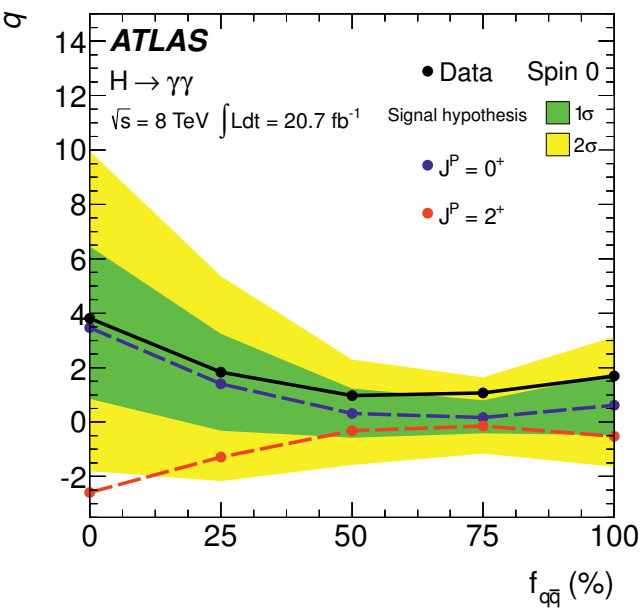

(b)

Figure 4. (a) Expected distributions of the test statistics $q \equiv \ln (L(0) / L(2))$ for the spin- 0 and spin-2 (produced by gluon fusion) hypotheses. The observed value is indicated by a vertical solid black line. The colored areas correspond to the integrals of the expected distributions used to compute the p-values for the rejection of each hypothesis [4]. (b) Median expected values of the test statistics for the spin- 0 (dashed blue line and corresponding $68 \%$ and $95 \%$ confidence level bands) and spin-2 (dashed red line) hypotheses, as well as the observed values (solid line) as a function of the fraction of $q \bar{q}$ production of the spin-2 signal $\left(f_{q \bar{q}}\right)$ [4].

production of the spin- 2 signal. The colored bands show the intervals corresponding to one and two standard deviations around the expectation for a spin-0 resonance.

\section{Differential cross section measurement}

Direct differential cross section measurements of the Higgs boson further elucidate its production and decay properties, and thus complement analyses of its couplings and spin mentioned in the previous two sections. The analysis is based on $20.3 \mathrm{fb}^{-1}$ of $8 \mathrm{TeV}$ data, which is the same dataset used in the spin analysis mentioned in Section 4 , but with a refined luminosity estimate and also updated data quality requirements. The event selection used here is also the same as the one used in the spin analysis described in Section 4.1.

The physics observables measured and reported here include: the transverse momentum $p_{\mathrm{T}}^{\gamma \gamma}$ and rapidity $\left|y^{\gamma \gamma}\right|$ of the Higgs boson, the jet multiplicity $N_{\text {jets }}$ in $H \rightarrow \gamma \gamma$ events, the azimuthal angle between the leading and the subleading jet $\Delta \phi_{j j}$, and the transverse component of the vector sum of the momenta of the Higgs boson and dijet system $p_{\mathrm{T}}^{\gamma \gamma j j}$.

The results are shown in Figure 5. The measured differential cross sections are compared with various theoretical predictions. 


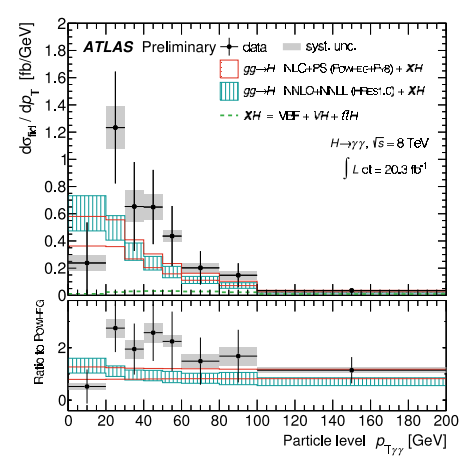

(a)

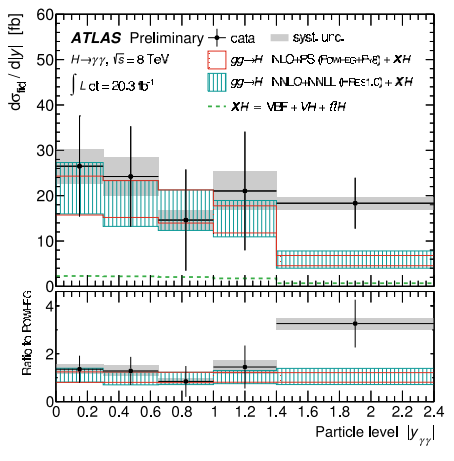

(b)

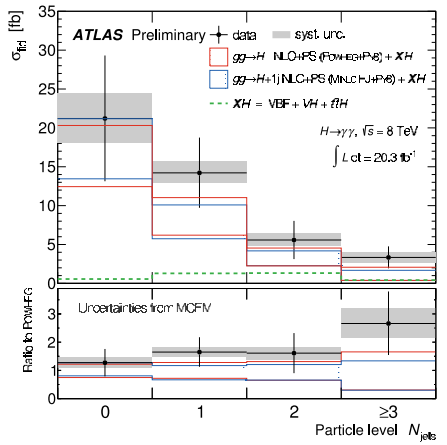

(c)

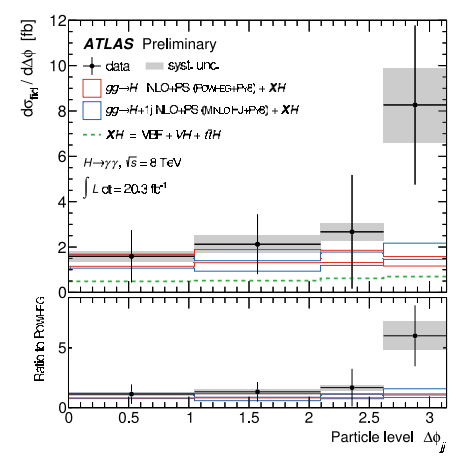

(d)

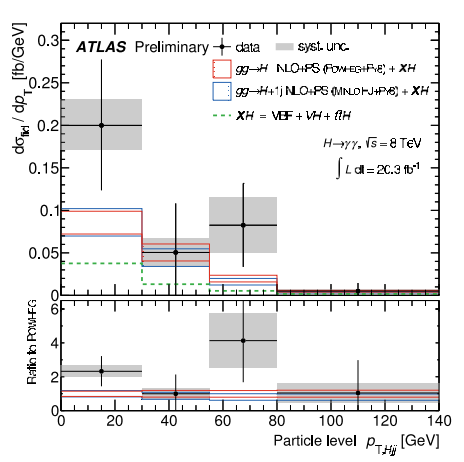

(e)

Figure 5. Observed differential cross sections of the Higgs bosons decaying into two isolated photons, for (a) $p_{\mathrm{T}}^{\gamma \gamma}$, (b) $\left|y^{\gamma \gamma}\right|$, (c) $N_{\mathrm{jets}}$, (d) $\Delta \phi_{j j}$, and (e) $p_{\mathrm{T}}^{\gamma \gamma j j}$. Systematic uncertainties are presented in grey solid box, and the black error bars represent the quadratic sum of statistical and systematic errors. The hatched histograms present theoretical predictions for the Standard Model at $\sqrt{s}=8 \mathrm{TeV}$ and $m_{H}=126.8 \mathrm{GeV}$. The sum of $V B F$ with $W H$, $Z H$, and $t \bar{t} H$ is denoted $X H$. These are added to the simulated $g g H$ predictions from POWHEG, MINLO, and HREs [5].

\section{Searches for $t \bar{t} H$ and FCNC $t \rightarrow c H$ decay}

\subsection{Search for $t \bar{t} H$ production mode}

While top quark-Higgs boson Yukawa coupling is a key ingredient for understanding EWSB, it can only be directly probed from the production of top-antitop quark pairs $(t \bar{t})$ in association with a Higgs boson $(t \bar{t} H)$. The search for $t \bar{t} H$ production in the $H \rightarrow \gamma \gamma$ decay channel is based on $20.3 \mathrm{fb}^{-1}$ of $8 \mathrm{TeV}$ data. The limits on the $t \bar{t} H$ production cross section relative to the expected SM cross section are shown in Figure 6(a). At $m_{H}=126.8 \mathrm{GeV}$ the observed limit is 5.3 times the SM cross section value, compared to an expected limit of 6.4 times the SM cross section value.

\subsection{Search for FCNC in $t \rightarrow c H$}

According to the SM, FCNC processes are forbidden at tree level and are very much suppressed at one-loop and higher orders due to the Glashow-Iliopoulos-Maiani mechanism. The search for $t \rightarrow c H$ 


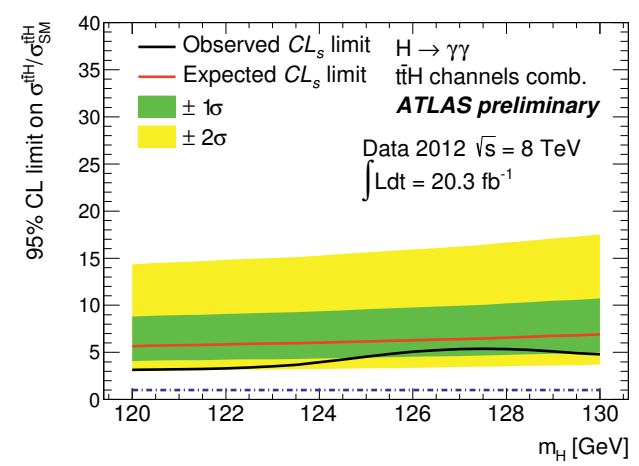

(a)

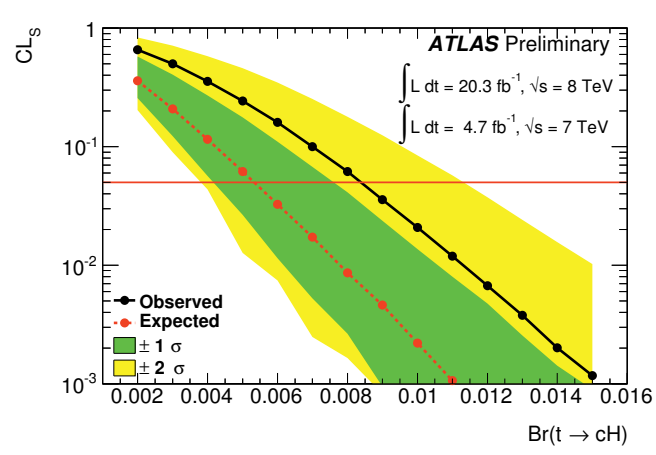

(b)

Figure 6. (a) Observed and expected 95\% confidence level upper limits on the $t \bar{t} H$ production cross section times the $H \rightarrow \gamma \gamma$ branching ratio divided by the SM expectations as a function of the Higgs boson mass. The contributions from all other Higgs boson production modes are set to the SM prediction taking into account their respective uncertainties [6]. (b) Evolution of $\mathrm{CL}_{\mathrm{S}}$ as a function of $\mathrm{Br}$ for the observation (full line) and the expectation in the absence of signal (dashed line). The bands at one and two $\sigma$ around the expected curve are also shown [7].

decay mode is carried out in the diphoton channel based on $4.7 \mathrm{fb}^{-1}$ of $7 \mathrm{TeV}$ data and $20.3 \mathrm{fb}^{-1}$ of $8 \mathrm{TeV}$ data. The observed (expected) limit on its branching ratio is set to be $0.83 \%(0.53 \%)$ at the $95 \%$ confidence level for $m_{H}=126.8 \mathrm{GeV}$, as shown in Figure 6(b).

\section{Conclusion}

We have presented results of the mass, coupling, spin, differential cross section measurements as well as the $t \bar{t} H$ and FCNC $t \rightarrow c H$ searches carried out in the $H \rightarrow \gamma \gamma$ channel. They are based on either the full data sample collected with the ATLAS detector in 2011 at $\sqrt{s}=7 \mathrm{TeV}$ and 2012 at $\sqrt{s}=$ $8 \mathrm{TeV}$, or only the full $8 \mathrm{TeV}$ data sample. Within the experimental and theoretical uncertainties, no significant deviation from the SM expectation is observed.

\section{References}

[1] ATLAS Collaboration, 2008 JINST 3 S08003.

[2] ATLAS Collaboration, ATLAS-CONF-2013-012, http://cds.cern.ch/record/1523698

[3] ATLAS Collaboration, Phys. Lett. B 726 (2013) 88

[4] ATLAS Collaboration, Phys. Lett. B 726 (2013) 120

[5] ATLAS Collaboration, ATLAS-CONF-2013-072, http://cds.cern.ch/record/1562925

[6] ATLAS Collaboration, ATLAS-CONF-2013-080, http://cds.cern.ch/record/1564319

[7] ATLAS Collaboration, ATLAS-CONF-2013-081, http://cds.cern.ch/record/1565103 